\title{
Odd Graceful Labeling Of Tensor Product Of Some Graphs
}

\author{
Usha Sharma, Rinku Chaudhary \\ Depatment Of Mathematics and Statistics \\ Banasthali University, Banasthali, Rajasthan-304022, India \\ rinkuchaudhary85@gmail.com
}

\begin{abstract}
A function $f$ is called an odd-graceful labeling of a graph $G=(V(G), E(G))$ with $p$ vertices and $q$ edges, if there exists an injection function .. with each edge uv assigned the label $\varnothing: \mathrm{V}(\mathrm{G}) \rightarrow\{0,1,2, \ldots ., 2 q-1\}$ the resulting edge labels are $\{1,3$, $5, \ldots . ., 2 q-1\}$. The tensor product of two graphs, $G$ and $H$, has a vertex set $V(G) \times V(H)$ and an edge between $(u, v)$ and $\left(u^{\prime}\right.$, $v^{\prime}$ ), iff both $u u^{\prime} \in E(G)$ and $v v^{\prime} \in E(H)$, here we denote tensor product by $\wedge$. In this paper, we prove $S_{n} \wedge S_{m}$ and $S_{n} \wedge P_{m}$ are odd graceful.
\end{abstract}

\section{Keywords}

Odd graceful labeling, Tensor product, Star Graph, Path.

\section{Academic Discipline And Sub-Disciplines}

Graph Theory

\section{SUBJECT CLASSIFICATION}

$05 \mathrm{C} 78$

\section{TYPE (METHOD/APPROACH)}

Odd graceful labeling, Tensor Product

\section{Council for Innovative Research}

\author{
Peer Review Research Publishing System
}

Journal: INTERNATIONAL JOURNAL OF COMPUTERS \& TECHNOLOGY

Vol 10, No 7

editor@cirworld.com

www.cirworld.com, member.cirworld.com 


\section{INTRODUCTION}

A graph $G$ consist of a set $V(G)$ of vertices and a set $E(G)$ of edges, where $E(G) \subseteq V(G) \times V(G)$. When a single graph $G$ is under consideration, we will write $V=V(G)$ for it's vertex set and $E=E(G)$ for it's edge set, furthermore, the order of $G$ is defined as cardinality of $V$ i.e $|V|$ denoted by $n$ often. If I a non negative integer $f(u)$ is assigned to each vertex $u$, then the vertices are said to be labeled. $G=(V, E)$ is itself a labeled graph if each edge e is given the value $f(e)=|f(u)-f(v)|$, where $u$ and $v$ are the end points of edge. Clearly every graph can be labeled in infinitely many ways. graph labeling is active research area in graph theory which has rigorous applications in coding theory, communication networks, optimal circuits layouts and graph decomposition problems. According to Beineke and Hegde [1] graph labeling serves as a frontier between number theory and the structure of graphs.

A function $f$ is called an odd-graceful labeling of a graph $G=(V, E)$ with $p$ vertices and $q$ edges, if there exists an injection function $\varnothing: V(G) \rightarrow\{0,1,2, \ldots, 2 q-1\}$ with each edge uv assigned the label $|f(u)-f(v)|$, the resulting edge labels are $\{1,3$, $5, \ldots, 2 q-1\}$. A graph which consist odd graceful labeling is odd graceful graph.

In 1991, Gnanajothi [3] proved the graph $C_{m} \times K_{2}$ is odd graceful iff $m$ even and the graph obtained from $P_{n} \times P_{2}$ by deleting an edge that joins to end points of the $P_{n}$ paths, this graph is known as the ladder graph. Author proved that every graph with an odd cycle is not odd graceful. This labeling has been studied in several articles. In 2000, Kathiresan [4] used the notation $P_{n ; m}$ to denote the graph obtained by identifying the end point of $m$ paths each has length $n$. Chawathe and Krishna [5] have extended the definition of odd gracefulness the countably infinite graphs and showed that all countably infinite bipartite graphs which are connected and locally finite have odd graceful labeling.

The path $P_{n}(n \geq 2)$ has vertices $v_{1}, v_{2}, \ldots, v_{n}$ and edges $v_{1} v_{2}, v_{2} v_{3}, \ldots, v_{n-1} v_{n}$. The cycle on $n$-vertices $C_{n}(n \geq 3)$ consists of $P_{n}$ plus an additional edge $\mathrm{v}_{1} \mathrm{v}_{\mathrm{n}}$; cycles are odd or even, according as the number of vertices is odd or even.

The $n$-dimensional star graph is denoted by $S_{n}$. The vertex set $V$ of $S_{n}$ is $\left\{a_{1}, \ldots, a_{n} \mid a_{1}, \ldots, a_{n}\right.$ is a permutation of $\left.1,2, \ldots ., n\right\}$ and the edge set $E$ is $\left\{\left(a_{1} a_{2}, \ldots, a_{i-1} a_{i} a_{i+1}, \ldots, a_{n}, a_{i} a_{2}, \ldots, a_{i-1} a_{1} a_{i+1}, \ldots, a_{n}\right) \mid a_{1}, \ldots, a_{n} \in V\right.$ and $\left.2 \leq i \leq n\right\}$. Clearly by definition, $S_{n}$ contains $n$ ! vertices and each vertex is of degree $(n-1)$.

The tensor product of two graphs $G$ and $H$ has a vertex set $V(G) \times V(H)$ and an edge between $(u, v)$ and $\left(u^{\prime}, v^{\prime}\right)$, iff both $u u^{\prime} \in E(G)$ and $v v^{\prime} \in E(H)$, here we denote tensor product by $\wedge$.

In this paper, we prove $S_{n} \wedge S_{m}$ and $S_{n} \wedge P_{m}$ are odd graceful.

\section{THE MAIN RESULTS}

Theorem 1. The tensor product of $S_{n}$ and $S_{m}$ i.e $S_{n} \wedge S_{m}$ is odd graceful.

Proof. Let $G=S_{n} \wedge S_{m}$ be a graph with $p$ vertices and q edges. The graph $G$ is obtained by tensor product of $S_{n}$ and $S_{m}$ where $n=1,2, \ldots, n$ and $m=1,2, \ldots, m$. $S_{n}$ has vertex set $\left\{u_{1}, u_{2}, \ldots, u_{n}\right\}$ and $S_{m}$ has $\left\{v_{1}, v_{2}, \ldots, v_{n}\right\}$. The graph $G$ has $q=2(n-$ 1) $(m-1)$ number of edges and $p=n . m$ number of vertices, as shown in figure:

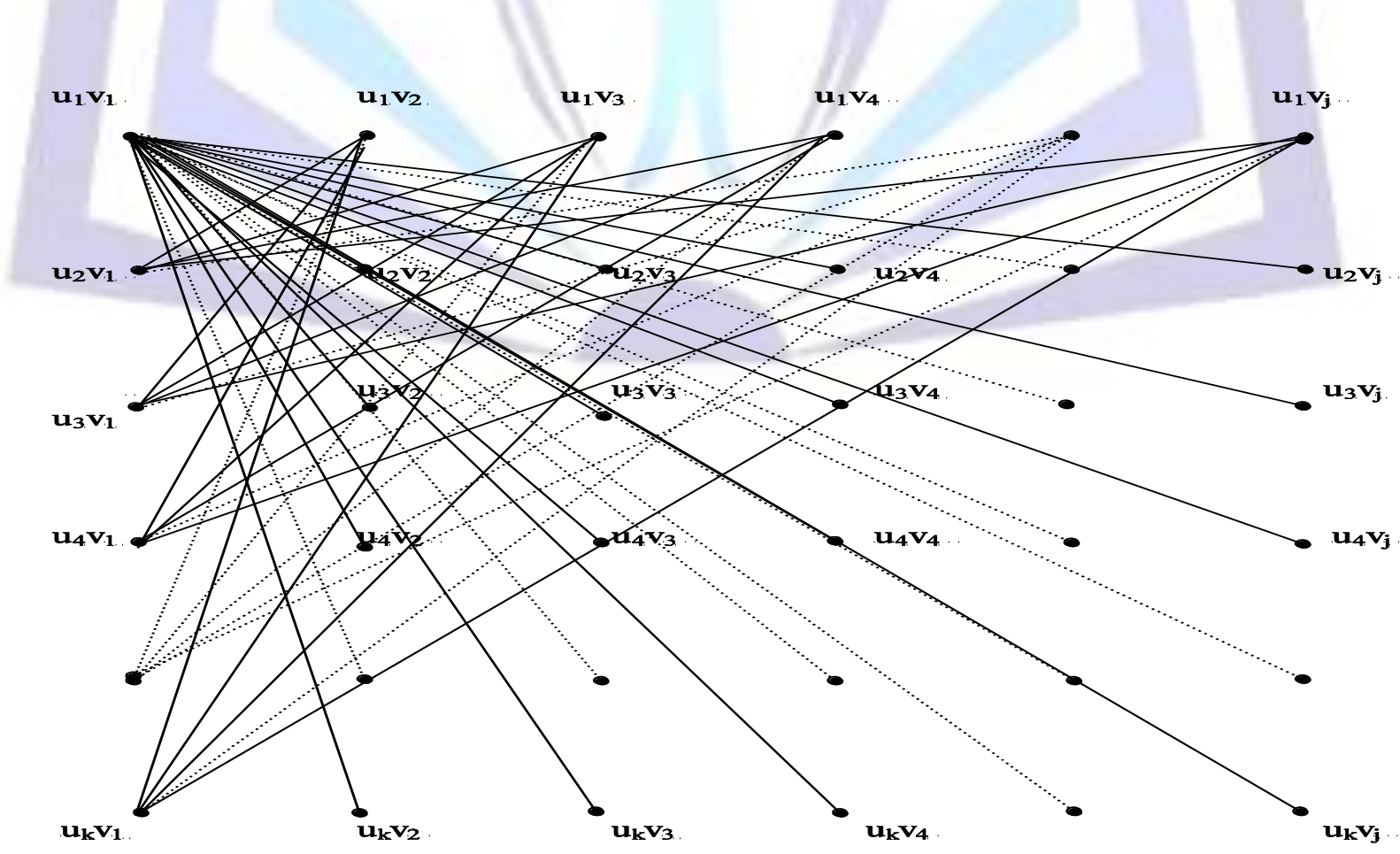

Fig 1: $S_{n} \wedge S_{m}$ 
Define $\varnothing: V(G) \rightarrow\{0,1,2, \ldots .,(2 q-1)\}$ as following:

$\varnothing\left(u_{1} v_{1}\right)=2$

$\varnothing\left(u_{k} v_{1}\right)=(2 q-1)-2(k-2)$

where, $2 \leq \mathrm{k} \leq \mathrm{n}$

$\varnothing\left(\mathrm{u}_{1} \mathrm{v}_{2}\right)=0$

$\varnothing\left(u_{k} v_{j}\right)=6(k-1)-3+2(j-2)+2(k-2)(m-4)$

where, $2 \leq \mathrm{k} \leq \mathrm{n}, 2 \leq \mathrm{j} \leq \mathrm{m}$

$\varnothing\left(u_{1} v_{j}\right)=2(n-1)(j-2)$,

$2 \leq \mathrm{j} \leq \mathrm{m}$

In accordance with the above labeling pattern the graph under consideration admits odd graceful labeling.

\section{Example :}

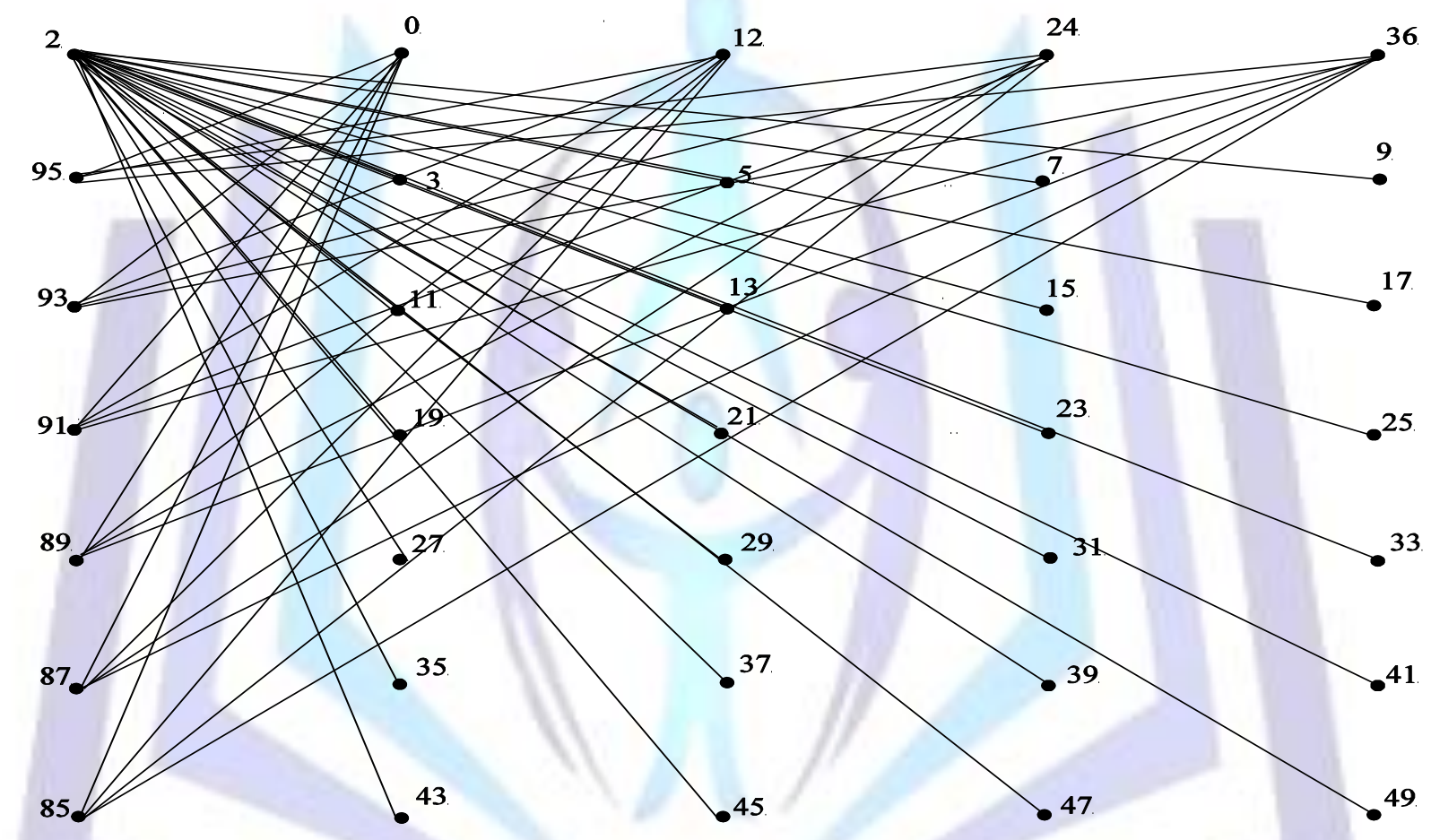

Fig 2: $S_{7} \wedge S_{5}$

Theorem 2. The tensor product of $S_{n}$ and $P_{m}$ i.e $S_{n} \wedge P_{m}$ is odd graceful.

Proof. Let $G=S_{n} \wedge P_{m}$ be a graph with p vertices and q edges. The graph $G$ obtained by tensor product of $S_{n}$ and $P_{m}$ where $n=1,2, \ldots, n$ and $m=1,2, \ldots, m$. $S_{n}$ has vertex set $\left\{u_{1}, u_{2}, \ldots, u_{n}\right\}$ and $P_{m}$ has vertex set $\left\{v_{1}, v_{2}, \ldots, v_{n}\right\}$. The graph $G$ has $q=2(n-1)(m-1)$ number of edges and $p=n . m$ number of vertices, as shown in figure:

Define $\varnothing: \mathrm{V}(\mathrm{G}) \rightarrow\{0,1,2, \ldots,(2 \mathrm{q}-1)\}$ as following: 


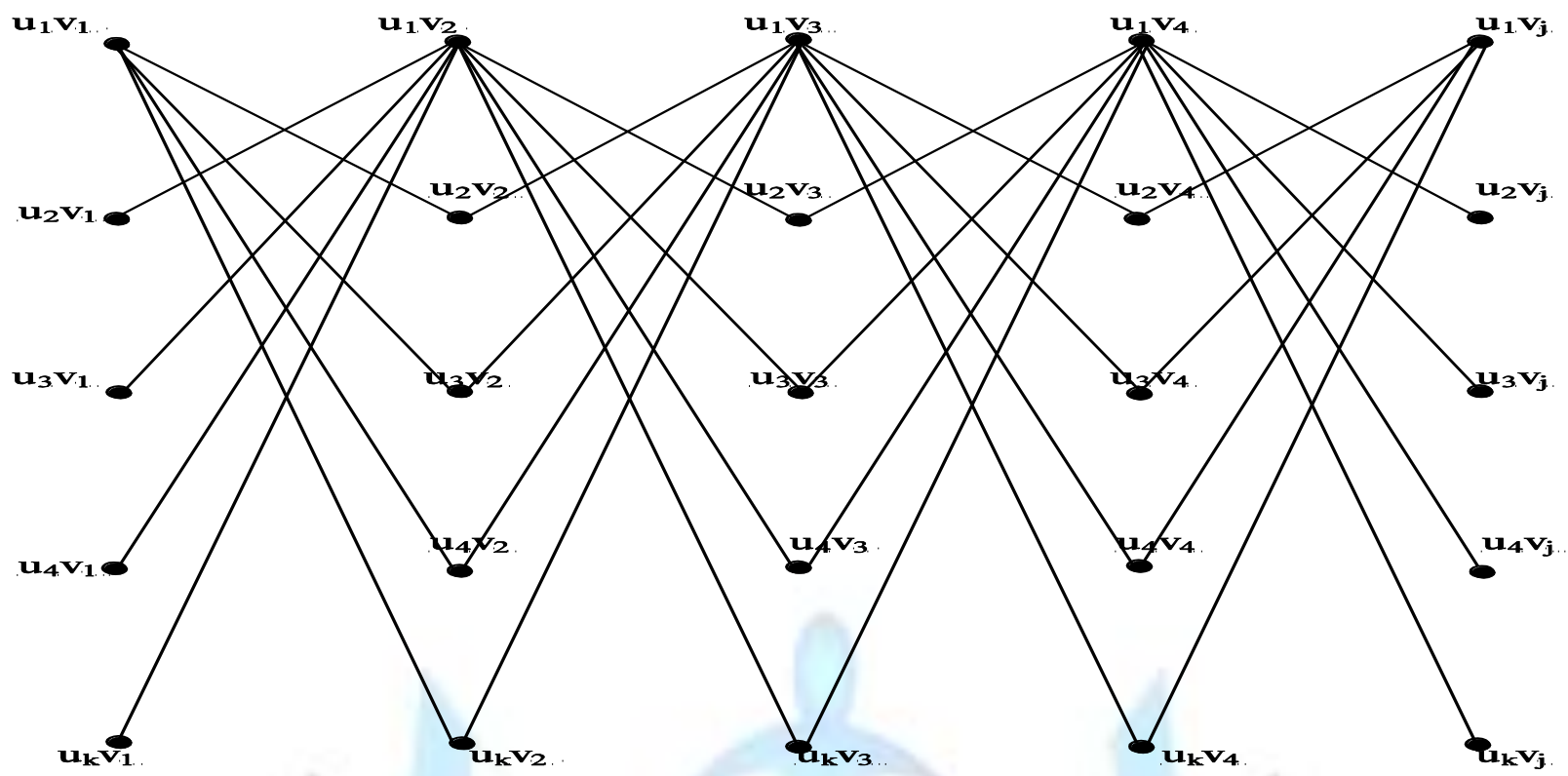

Fig 3: $S_{n} \wedge P_{m}$

$\varnothing\left(u_{k} v_{1}\right)=2(k-1)$

$\varnothing\left(u_{1} v_{2}\right)=1$

$\varnothing\left(u_{1} v_{2 j+1}\right)=2(n-1)(j+1)$

$\varnothing\left(u_{1} v_{2 j+2}\right)=2(n-1)(j+1)+1$,

$\varnothing\left(\mathrm{u}_{\mathrm{k}} \mathrm{v}_{2}\right)=(2 \mathrm{q}-1)-2(\mathrm{k}-1)$,

$\varnothing\left(\mathrm{u}_{\mathrm{k}} \mathrm{V}_{2 \mathrm{j}+1}\right)=(2 \mathrm{q}-1)-2(\mathrm{k}-2)-2(\mathrm{n}-1)-6(\mathrm{n}-1)(\mathrm{j}-1)+1$,

$\varnothing\left(u_{k} v_{2}\right)=(2 q-1)-2(k-2)-2(n-1)-6(n-1)(j-1)+1-(2 n-1)$,

where, $1 \leq \mathrm{k} \leq \mathrm{n}$

In accordance with the above labeling pattern the graph under consideration admits odd graceful labeling.

\section{Example}

:

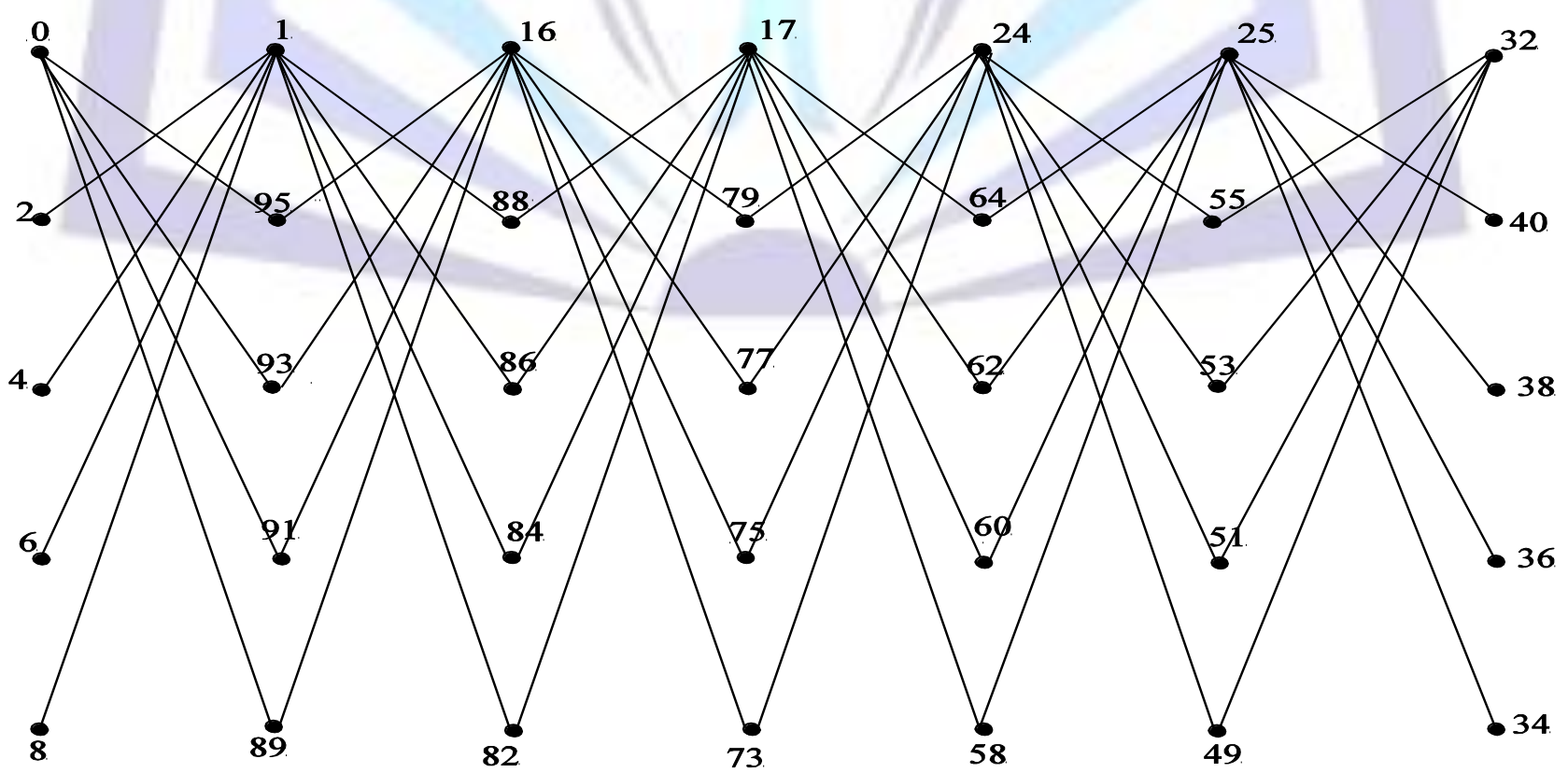

Fig 4: $S_{5} \wedge P_{7}$ 


\section{CONCLUSION}

We have given a systematic approach to find odd graceful labeling of tensor products $S_{n} \wedge S_{m}$ and $S_{n} \wedge P_{m}$ and so these are odd graceful graphs.

\section{ACKNOWLEDGMENTS}

Ms RINKU CHAUDHARY, one of the authors of this paper acknowledges the grant received from Department of Science \& Technology (D.S.T.), Government of India, New Delhi for carrying out this research.

\section{REFERENCES}

[1] Beineke, L. W. and Hegde, S. M. 2001. Strongly Multiplicative Graphs, Discuss. Math. Graph Theory , 2(2001)-63-75.

[2] Chawathe, P. D. and Krishna, V., Odd Graceful Labelings of Countably Infinite Locally Finite Bipartite Graphs, Con-ference on Graph Theory and its Applications, March 2001, School of Mathematics, Anna University, Chennai.

[3] Gallian, J.A., A Dynamic Survey of Graph Labeling, The Electronic Journal of Combinotrics, 18(2011) DS6.

[4] Gnanajothi, R.B. 1991. Topics in Graph Theory, Ph.D Thesis, Madurai Kamaraj University, Madurai.

[5] Kathiresan, K. 2000. Two Classes of Graceful Graphs, Ars. Combin.55, 129-132.

\section{Author's biography with Photo}

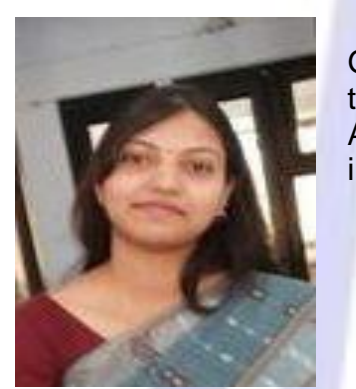

Usha Sharma has completed her PhD degree on the topic QUALITY OF SERVICE: ISSUE K GRAPH THEORTIC SOLUTION from Banasthali University(India). She was working as JRF under the DST project Center of Mathematical Sciences at Banasthali University. Now, she is working as Assistant Proffessor in Depatment of Mathematics \& Statistics in Banashali University. Her main interest include wireless sensor networks, labeling of graphs, dominating sets in graphs.

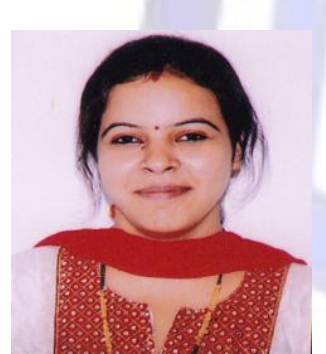

Rinku Chaudhary is a PhD student in Banasthali University(India). She received her M.Phil.(2011) in Mathematical Sciences at the same university. She is working as JRF under the DST project Center of Mathematical Sciences at Banasthali University. Her main interest include wireless sensor networks, labeling of graphs, coloring of graphs. 